LA-UR-02-5546

Approved for public release; distribution is unlimited.

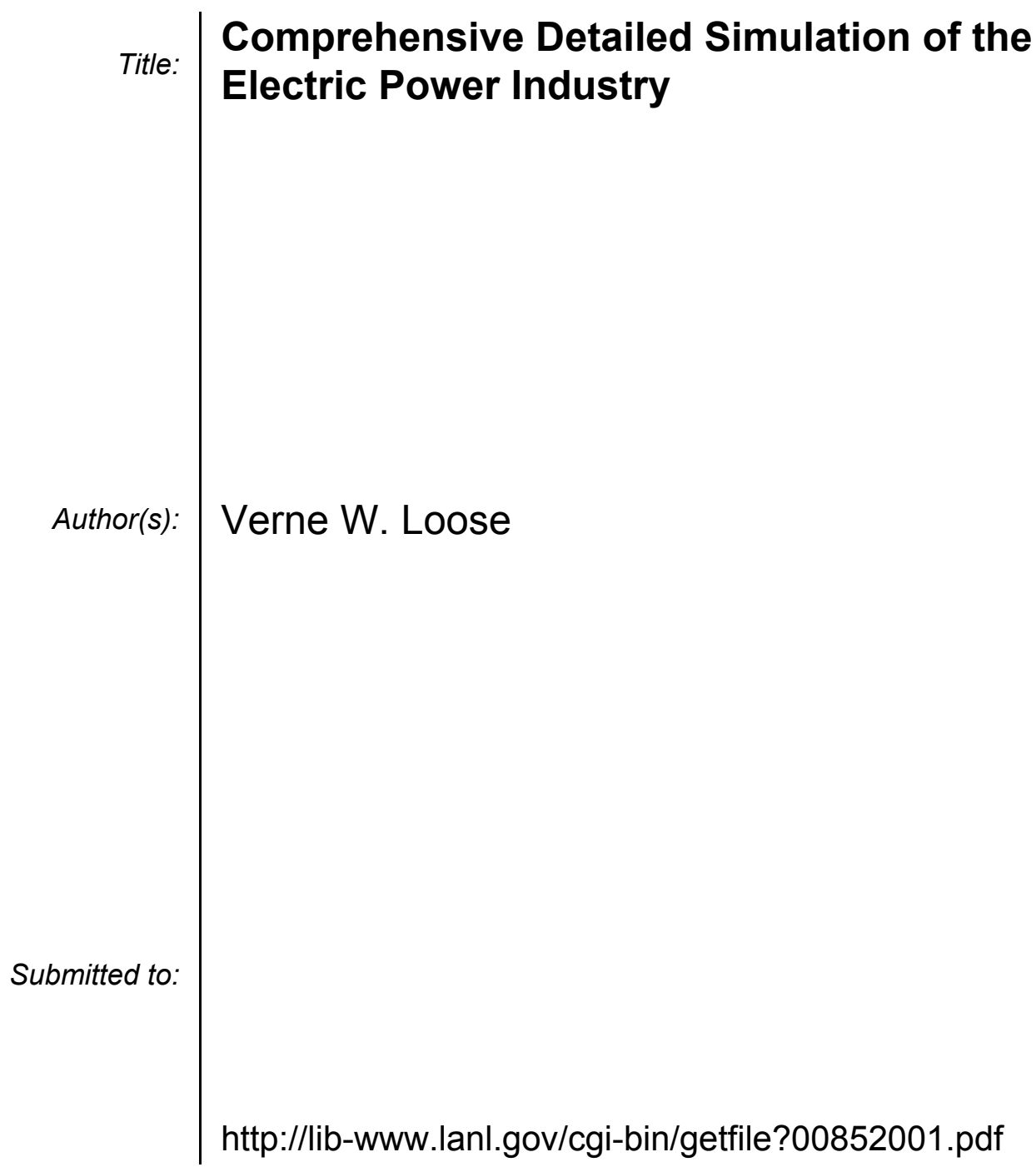

Los Alamos National Laboratory, an affirmative action/equal opportunity employer, is operated by the University of California for the U.S. Department of Energy under contract W-7405-ENG-36. By acceptance of this article, the publisher recognizes that the U.S. Government retains a nonexclusive, royaltyfree license to publish or reproduce the published form of this contribution, or to allow others to do so, for U.S. Government purposes. Los Alamos National Laboratory requests that the publisher identify this article as work performed under the auspices of the U.S. Department of Energy. Los Alamos National Laboratory strongly supports academic freedom and a researcher's right to publish; as an institution, however, the Laboratory does not endorse the viewpoint of a publication or guarantee its technical correctness. 


\title{
Comprehensive Detailed Simulation of the Electric Power Industry
}

\author{
Verne W. Loose*,
}

\begin{abstract}
A model of the Western Systems Coordinating Council electric generation and transmission system in eleven western states and portions of Canada and Mexico was constructed and several market protocol simulations were run. Results demonstrated the efficiency of energy trading between ISOs with low cost electric capacity could increase reliability and save money.
\end{abstract}

\section{Background and Research Objectives}

The Electric Industry Simulation System (ELECTRISIMS) project aims to research and develop a comprehensive, detailed simulation of the electric power industry:

- Comprehensive in that we will include the whole North American continent because that natural limit is becoming the scale of tight interconnection.

- Detailed in that we will include each significant element at the level of generators, transmission elements, varied control elements, and load distribution buses.

- Industry in that we will include the regulatory, financial, and market entities that interact with the engineering elements.

\section{Importance to LANL's Science and Technology Base and National R\&D Needs}

Qualitatively there are four areas of invention required in meeting our goal: (1) Given the very large sparse matrix problem implied, develop efficient solution techniques for the ASCI environment; (2) Develop software operators to reduce the scope of the problem consistently with applications, including reductions to mixed resolution applications; (3) Give n assorted massive data bases, develop software to calculate the required matrix elements, and (4) Develop synthetic demand models and market models which (along with the established electrical engineering models) enable filling out the data bases.

\section{Scientific Approach and Accomplishments}

\section{Electric Power Industry Simulation System (ElectriSim)}

Goals. The goal of the ElectriSim project was to develop a comprehensive, detailed simulation of the electric industry. The simulation would be:

- Comprehensive in that we propose including the whole North American continent because that is becoming the scale of tight interconnection.

- Detailed in that we propose to include each significant element at the level of generators, transmission, varied control elements, and load distribution buses. Detailed also in that market participants whose decisions over operating criteria may be driven largely by profit motives will control each of these physical elements.

\footnotetext{
*1oose@lanl.gov
} 
- Industry in that we intend to include the regulatory, financial, and market factors that interacts with the engineering elements.

Our vision was that policy makers and other users to conduct analyses of policies and market rules could use the simulation and protocols that would help guide the industry in its evolution to market competition.

First Year. We developed the technical approach and a research plan that would lead to staged development of the model we envisioned. This encompassed development of an architecture for the simulation that would include the integration of the physical elements of the system and the agents that would be the elements of the market, together with the method for integration of the two very different systems. We obtained a power flow code that we intended to parallelize for running on the ASCI machines and did considerable work to plan and execute the installation. It was our intent that this code could provide the basis for producing the largest, fastest power flow code running a problem in excess of 100,000 nodes. We developed a simple prototype model to illustrate and characterized the integration of the marketplace with the physical infrastructure. This prototype was based on a "stock-market" type model and included several novel features among them increasing cost of transmission in conjunction with increased flow along the transmission lines.

Second Year. Achievements during the first year led to the establishment of a CRADA with the California Independent System Operator (Cal-ISO) for performing joint analysis using the models and approach we developed. When funding for the project was cut in November 1999 it was determined that remaining funding was sufficient only to conduct the CRADA work in support of the Cal-ISO. Hence all effort was directed toward that end. During this year we worked with the Cal-ISO to define a problem that was of interest to both entities. The result was an examination, in the context of ElectriSimS, of the benefits that would accrue from establishing a regional transmission organization throughout the Western Systems Coordinating Council Region and a comparison of two market rule sets that might govern markets in this large territory. The remainder of the year involved structuring the simulation to address the problem and acquisition of the data necessary to populate the model. The graphic above presents a schematic of the structure of the model used for the Cal-ISO CRADA analysis. Also during this second year, ElectriSimS models and team members collaborated with the Urban Security LDRD project and produced some very interesting analyses of earthquake consequences for the electric power network in the Los Angeles basin.

Third Year. In this the third and final year of the project we are working to complete and report the analysis we have conducted with the Cal-ISO and preparing to close out the CRADA. We have completed the computer simulations on the ROCKHOPPER Linux cluster and have drafted a report that is now in the hands of our Cal-ISO counterparts for review. We anticipate receiving their input to this joint report in the very near future.

An additional activity has involved the team in an effort to establish programmatic funding for ElectriSimS. We have developed a 5-year plan during which the development process would be accomplished. While our efforts to date have not borne fruit, we are hopeful that funding to continue our effort will be identified. Our resolve that a simulation capability such as ElectriSimS is needed is reinforced by events in California. It is clear from these events during that restructuring electric markets is not an easy policy to implement and that very serious unintended consequences can flow from well-intentioned policies. We believe that our vision for ElectriSimS has been vindicated by these events and reactions to the situation. 
Products. Many products have flowed from the effort. There are several models together with data to populate them that are now extant all of which could be used for additional analyses. We have a successful relationship with an organization that could be important to additional work in the area-the California ISO. There are a number of technical papers and reports that have been produced and presented at conferences and submitted to peer-reviewed journals.

Assessment. The ElectriSimS LDRD effort has been successful in that a significant portion of our initial vision has been realized. We have shown the feasibility of dynamically integrating market and physical models of electric power networks. While we have not yet been able to realize the full vision for a fully developed market model, much of the groundwork toward this end has been laid. Existing efforts within D Division may pick up from where we left off and complete the vision.

\section{Publications}

L. Jonathan Dowell, Dale B. Henderson, Verne W. Loose, Douglas J. Roberts, Kenneth A. Werley, LANL, Vicken Kasarjian, California Independent System Operator, Alex Papalexopoulos, ECCO International.

"Joint Project On California Electric Market and Grid Modeling: Final Report," Summer 2001. LA-CP-01-440.

\section{J. Dowell and S. Maheshwari}

"Simulating Earthquake Damage to the Electric-Power Infrastructure:

A Case Study for Urban Planning and Policy Development"

(LA-UR-00-3777)

(Submitted for publication in a volume summarizing the work of the International Decade on Natural Disaster Reduction, by Profs. Rodrigue and Rovai, California State University, in press)

L. J. Dowell, D. J. Roberts, and D. B. Henderson

"On Solving Nearly-Singular, Sparse Systems of Linear Equations:

Diakoptics Techniques for Parallel Computing"

(LA-UR-00-2175)

(submitted to Applied Computing Review, still being reviewed)

V. W. Loose and L. J. Dowell

"Economic and Engineering Constraints on the Restructuring of the Electric-Power Industry: A Consideration of Reactive-Power Ancillary Services"

(LA-UR-00-2230)

(INFORMS, Salt Lake City, 2000)

S. Maheshwari and L. J. Dowell

"Elysian Park (Los Angeles) Earthquake Simulation:

Impacts on Electric-Power Infrastructure"

(LA-UR-99-4429)

(presented without publication at Urban and Regional Information Systems Association, Chicago, 1999)

L. J. Dowell and D. B. Henderson

"A Comprehensive, Detailed Simulation of the Electric-Power Industry: Harnessing the Los Alamos National Laboratory High-Performance Computing Infrastructure" 
(LA-UR-98-5920)

(Proceedings of the High Performance Computing Symposium, SCS, San Diego, 1999)

L. J. Dowell

"Estimation of the Service Areas of Electric-Power Substations by Cellular Automata" (LA-UR-98-5769)

(Proceedings of the High Performance Computing Symposium, SCS, San Diego, 1999)

\section{References}

N/A

\section{Figure Captions}

N/A 